\title{
Smoothing Spline Estimation of Variance Functions
}

\author{
Anna LiU, Tiejun Tong and Yuedong WANG* \\ January 11, 2006
}

\begin{abstract}
This article considers spline smoothing of variance functions. We focus on selection of smoothing parameters and develop three direct data-driven methods: unbiased risk (UBR), generalized approximate cross validation (GACV) and generalized maximum likelihood (GML). In addition to guaranteed convergence, simulations show that these direct methods perform better than existing indirect UBR, generalized cross validation (GCV) and GML methods. The direct UBR and GML methods perform better than the GACV method. An application to array-based comparative genomic hybridization data illustrates the usefulness of the proposed methods.

KEY WORDS: array-based comparative genomic hybridization; generalized approximate cross validation; generalized maximum likelihood; heteroscedasticity; smoothing parameter; unbiased risk.
\end{abstract}

\section{INTRODUCTION}

Modeling local variability in terms of variance function is an important problem with a wide range of applications. For example, variance function estimates are needed in finance, quality control and immunoassay for measuring volatility or risk (Andersen and Lund 1997, Gallant and Tauchen 1997), experimental design (Box 1988), prediction (Carroll 1987, Yao and Tong 1994) and calibration (Raab 1981, Watters, Carroll and Spiegelman 1987). Variance estimation

*Anna Liu is Assistant Professor, Department of Mathematics and Statistics, University of Massachusetts, Amherst, MA 01003 (email: anna@math.umass.edu); Tiejun Tong is Postdoctoral Associate, Department of Epidemiology and Public Health, Yale University, New Haven, CT 06520 (email: tiejun.tong@yale.edu); and Yuedong Wang is Professor, Department of Statistics and Applied Probability, University of California, Santa Barbara, California 93106 (email: yuedong@pstat.ucsb.edu). This research was supported by NIH Grant R01 GM58533. Address for correspondence: Yuedong Wang, Department of Statistics and Applied Probability, University of California, Santa Barbara, California 93106. 
is especially important for detecting genes with differential expression across experimental conditions based on microarray data (Huang and Pan 2002, Wang and Guo 2004). With a small number of replicated array experiments, the standard estimates of variances are unreliable. Various methods have been proposed to improve estimation of the variances which usually leads to more powerful tests (Huang and Pan 2002, Wang and Guo 2004, Cui, Hwang, Qiu, Blades and Churchill 2005). We will apply our variance function estimation methods to array-based comparative genomic hybridization $(\mathrm{aCGH})$ data in Section 5. More applications of variance function estimation can be found in Carroll and Ruppert (1988).

Research on nonparametric estimation of variance functions has attracted a great deal of attention (Carroll 1982, Silverman 1985, Hall and Carroll 1989, Ruppert, Wand, Holst and Hssjer 1997, Fan and Yao 1998, Yuan and Wahba 2004, Dai and Guo 2005). Most research concentrates on heteroscedastic regression. Both local polynomial smoothers and smoothing splines were used to model the variance function nonparametrically. Within the smoothing spline framework, Yuan and Wahba (2004) used the generalized approximate cross validation (GACV) method to select the smoothing parameter for estimating the variance function, while Dai and Guo (2005) treated squared pseudo-residuals (lag-one differences) as Gaussian data. The main goal of this paper is to develop and compare several data-driven smoothing parameter selection methods for the smoothing spline estimation of variance functions.

To simplify exposition, we focus on the situation when direct observations on a variance function are available. Specifically, we have independent observations $\left\{\left(x_{i}, y_{i}\right), i=1, \cdots, n\right\}$ where

$$
y_{i}=\exp \left(f\left(x_{i}\right)\right) \chi_{i, k}^{2} / k
$$

$\chi_{i, k}^{2}$ are iid Chi-square random variables with degree of freedom $k$. Our goal is to estimate the variance function $f$ nonparametrically.

One typical situation leading to (1) is the following heteroscedastic regression model with replicates

$$
z_{i j}=\mu_{i}+\exp \left(f\left(x_{i}\right) / 2\right) \epsilon_{i j}, \quad i=1, \cdots, n ; j=1, \cdots, k+1,
$$

where $\epsilon_{i j} \stackrel{i i d}{\sim} N(0,1)$. Then the sample variances $y_{i}=\sum_{j=1}^{k+1}\left(z_{i j}-\sum_{j=1}^{k+1} z_{i j} /(k+1)\right)^{2} / k$ follow the model (1). See Section 5 and Raab (1981) for real examples of the model (2).

Most nonparametric methods for heteroscedastic regression use squared residuals after fitting a nonparametric model to the mean function (Silverman 1985, Hall and Carroll 1989, Ruppert et al. 1997, Fan and Yao 1998, Yuan and Wahba 2004), or squared pseudo-residuals after removing the mean by differences (Müller and Stadtmüller 1987, Fan and Yao 1998, Dai and Guo 2005), as observations on a variance function. Under suitable conditions, these squared residuals and pseudo-residuals follow the model (1) asymptotically (Fan and Yao 1998, Dai and 
Guo 2005). The degree of freedom $k$ equals one for most of these methods. Therefore, results in this article also shed light on selection of smoothing parameters for these methods when $k$ is small.

We introduce smoothing spline models and an estimation procedure in Section 2. In Section 3 , we review some existing indirect methods and propose three direct methods for estimating smoothing parameters. We report results of extensive simulations in Section 4 and apply our methods to a real aCGH data in Section 5.

\section{SMOOTHING SPLINE MODELS FOR VARIANCE FUNCTIONS}

We assume the model (1) in the reminder of this paper. Consider the Chi-square distribution as a special case of the Gamma distribution, we have $y_{i} \sim \operatorname{Gamma}\left(k / 2,2 \exp \left(f\left(x_{i}\right)\right) / k\right)$ with log-likelihood

$$
l_{i}\left(f_{i}\right)=-k y_{i} \exp \left(-f_{i}\right) / 2-k f_{i} / 2+c\left(y_{i}\right),
$$

where $f_{i}=f\left(x_{i}\right)$ and $c\left(y_{i}\right)=\log \left(y_{i}^{k / 2-1}(k / 2)^{k / 2} / \Gamma(k / 2)\right)$ which is independent of $f_{i}$. Spline smoothing methods for the exponential family in Wahba, Wang, Gu, Klein and Klein (1995) can be employed to estimate the function $f$. Note, however, instead of the canonical link (reciprocal) used in the previous publications, we use the logarithmic link in this paper to free the positive constraint on the variance function. We will also propose new direct methods for selecting smoothing parameters.

For simplicity, we assume that the domain of the function $f$ is $[0,1]$ and $f$ belongs to the reproducing kernel Hilbert space (RKHS)

$$
W_{m}([0,1])=\left\{f: f, f^{\prime}, \cdots, f^{(m-1)} \text { absolutely continuous, } \int_{0}^{1}\left(f^{(m)}\right)^{2} d x<\infty\right\} .
$$

Our methods apply to general smoothing spline and smoothing spline ANOVA models (Wahba et al. 1995). The smoothing spline estimate of $f, f_{\lambda}$, is the minimizer of the penalized likelihood

$$
-\sum_{i=1}^{n} l_{i}\left(f_{i}\right)+\frac{n \lambda}{2} \int_{0}^{1}\left(f^{(m)}\right)^{2} d x
$$

where $\lambda$ is a smoothing parameter controlling the trade-off between the goodness-of-fit and the smoothness of the function. Let $B_{r}$ be Bernoulli polynomials, $\phi_{r}(x)=B_{r-1}(x) /(r-1)$ !, $r=1, \cdots, m$, and $R_{1}(s, t)=\phi_{m+1}(s) \phi_{m+1}(t)+(-1)^{m-1} \phi_{2 m+1}(s-t)$. Then the solution to $(5)$ is (Wahba et al. 1995, Gu 2002)

$$
f_{\lambda}(x)=\sum_{i=1}^{m} d_{i} \phi_{i}(x)+\sum_{i=1}^{n} c_{i} R_{1}\left(x_{i}, x\right)
$$


For any fixed $\lambda$, coefficients $\boldsymbol{c}=\left(c_{1}, \cdots, c_{n}\right)^{T}$ and $\boldsymbol{d}=\left(d_{1}, \cdots, d_{m}\right)^{T}$ can be solved by the Newton procedure (Wahba et al. 1995). Let $u_{i}=-d l_{i}\left(f_{i}\right) / d f_{i}=-k y_{i} \exp \left(-f_{i}\right) / 2+k / 2$ and $w_{i}=-d^{2} l_{i}\left(f_{i}\right) / d f_{i}^{2}=k y_{i} \exp \left(-f_{i}\right) / 2$. The Newton procedure updates $\boldsymbol{c}$ and $\boldsymbol{d}$ by iteratively reformulating the minimization problem (5) as

$$
\sum_{i=1}^{n} w_{i-}\left(\tilde{y}_{i}-f_{i}\right)^{2}+n \lambda \int_{0}^{1}\left(f^{(m)}\right)^{2} d x
$$

where $\tilde{y}_{i}=f_{i_{-}}-u_{i_{-}} / w_{i-}$ is the pseudo-data, the subscript minus denotes quantities evaluated at the previous Newton iteration (Gu 1992, Wahba et al. 1995).

\section{METHODS FOR SELECTING SMOOTHING PARAMETERS}

\subsection{Indirect Methods}

A good choice of the smoothing parameter $\lambda$ is crucial to the performance of the smoothing spline estimate $f_{\lambda}$. Note that $(7)$ is the penalized weighted least squares for the working variables $\tilde{y}_{i}$ and working weights $w_{i-}$. An indirect (or iterative) method chooses a smoothing parameter at each iteration for the reformulated problem (7) and hopes it will converge $(\mathrm{Gu}$ 1992). To estimate $\lambda$ at each iteration, we may use the UBR, GCV or GML methods which respectively minimizes the following UBR, GCV and GML scores

$$
\begin{aligned}
U_{-}(\lambda) & =\frac{1}{n}\left\|(\boldsymbol{I}-\boldsymbol{A}(\lambda)) \boldsymbol{W}_{-}^{1 / 2} \tilde{\boldsymbol{y}}\right\|^{2}+2 \frac{\hat{\sigma}^{2}}{n} \operatorname{tr} \boldsymbol{A}(\lambda), \\
V_{-}(\lambda) & =\frac{1 / n\left\|(\boldsymbol{I}-\boldsymbol{A}(\lambda)) \boldsymbol{W}_{-}^{1 / 2} \tilde{\boldsymbol{y}}\right\|^{2}}{[(1 / n) \operatorname{tr}(\boldsymbol{I}-\boldsymbol{A}(\lambda))]^{2}}, \\
M_{-}(\lambda) & =\frac{\tilde{\boldsymbol{y}}^{T} \boldsymbol{W}_{-}^{1 / 2}(\boldsymbol{I}-\boldsymbol{A}(\lambda)) \boldsymbol{W}_{-}^{1 / 2} \tilde{\boldsymbol{y}}}{\left[\operatorname{det}^{+}\left(\boldsymbol{W}_{-}^{1 / 2}(\boldsymbol{I}-\boldsymbol{A}(\lambda)) \boldsymbol{W}_{-}^{1 / 2}\right)\right]^{\frac{1}{n-m}}},
\end{aligned}
$$

where $\tilde{\boldsymbol{y}}=\left(\tilde{y}_{1}, \cdots, \tilde{y}_{n}\right)^{T}, \boldsymbol{W}_{-}=\operatorname{diag}\left(w_{1-}, \cdots, w_{n-}\right), f_{\lambda i}=f_{\lambda}\left(x_{i}\right), \boldsymbol{A}(\lambda)$ satisfies

$\left(w_{1-}^{1 / 2} f_{\lambda 1}, \cdots, w_{n-}^{1 / 2} f_{\lambda n}\right)^{T}=\boldsymbol{A}(\lambda)\left(w_{1-}^{1 / 2} \tilde{y}_{1}, \cdots, w_{n-}^{1 / 2} \tilde{y}_{n}\right)^{T}, \hat{\sigma}^{2}=\sum_{i=1}^{n} u_{i-}^{2} / n w_{i-}$, and det ${ }^{+}$is the product of the nonzero eigenvalues (Wahba et al. 1995, Gu 2002).

One problem with the indirect methods is that it does not guarantee convergence. For Binomial and Poisson data, extensive simulations indicate that convergence is achieved for almost all situations (Wang, Wahba, Chappell and Gu 1995). The performance of these indirect methods for Gamma data has not been studied. For Binomial and Poisson data, some direct methods have been developed and found to work better than the indirect methods (Xiang and Wahba 1996, Gu and Xiang 2001, Yuan 2005). Again, direct methods for Gamma data have not been developed.

One measure of the discrepancy between the spline estimate $f_{\lambda}$ and the true function $f$ is 
the Kullback-Leibler distance (Wahba et al. 1995)

$$
K L\left(f, f_{\lambda}\right)=\frac{1}{n} \sum_{i=1}^{n} \mathrm{E}_{f_{i}}\left(l_{i}\left(f_{i}\right)-l_{i}\left(f_{\lambda i}\right)\right)=\frac{k}{2 n} \sum_{i=1}^{n}\left(\exp \left(f_{i}-f_{\lambda i}\right)+f_{\lambda i}\right)-\frac{k}{2 n} \sum_{i=1}^{n}\left(1+f_{i}\right) .
$$

$U_{-}(\lambda)$ provides a proxy of $K L\left(f, f_{\lambda}\right)$ (Wang et al. 1995). Ignoring the last term in (11) which is independent of $\lambda$ and the multiplying constant $k / 2$, we consider the comparative KullbackLeibler criterion

$$
C K L\left(f, f_{\lambda}\right)=\frac{1}{n} \sum_{i=1}^{n}\left(\exp \left(f_{i}-f_{\lambda i}\right)+f_{\lambda i}\right)
$$

One approach is to find $\lambda$ such that $C K L\left(f, f_{\lambda}\right)$ is minimized. However, as other loss functions, $C K L\left(f, f_{\lambda}\right)$ cannot be minimized directly since $f$ is unknown. In the following three subsections, we propose three direct data-driven methods to select $\lambda$.

\subsection{Unbiased Risk Method}

In this subsection we derive an unbiased estimate of $\mathrm{E}\left(C K L\left(f, f_{\lambda}\right)\right)$. Let $h_{\lambda}(i, z, \cdot)$ be the minimizer of (5) when the $i$ th observation, $y_{i}$, is replaced by $z$. Let $g_{\lambda i}(z)=h_{\lambda}\left(i, z, x_{i}\right)$ and $v(t)=\int_{0}^{t} \exp \left(-g_{\lambda i}(z)\right) z^{k / 2-1} d z$. Then for any fixed $\boldsymbol{y}_{-i}=\left(y_{1}, \cdots, y_{i-1}, y_{i+1}, \cdots, y_{n}\right)^{T}$, when $k \geq 3$, we have

$$
\begin{aligned}
& \mathrm{E}\left(\exp \left(f_{i}-f_{\lambda i}\right)\right) \\
= & \frac{\exp \left(f_{i}\right)}{\Gamma(k / 2)}\left(\frac{2 \exp \left(f_{i}\right)}{k}\right)^{-\frac{k}{2}} \int_{0}^{\infty} \exp \left(-g_{\lambda i}(t)\right) t^{\frac{k}{2}-1} \exp \left(\frac{-k t}{2 \exp \left(f_{i}\right)}\right) d t \\
= & \frac{\exp \left(f_{i}\right)}{\Gamma(k / 2)}\left(\frac{2 \exp \left(f_{i}\right)}{k}\right)^{-\frac{k}{2}}\left\{\left.v(t) \exp \left(\frac{-k t}{2 \exp \left(f_{i}\right)}\right)\right|_{0} ^{\infty}-\int_{0}^{\infty} v(t) d\left(\exp \left(\frac{-k t}{2 \exp \left(f_{i}\right)}\right)\right)\right\} \\
= & \frac{k}{2 \Gamma(k / 2)}\left(\frac{2 \exp \left(f_{i}\right)}{k}\right)^{-\frac{k}{2}} \int_{0}^{\infty} v(t) \exp \left(\frac{-k t}{2 \exp \left(f_{i}\right)}\right) d t \\
= & \frac{k}{2} \mathrm{E}\left(v\left(y_{i}\right) y_{i}^{-(k / 2-1)}\right)
\end{aligned}
$$

where we used the facts that $\mathrm{E}\left(\exp \left(-f_{\lambda i}\right)\right)$ exists and $\left.v(t) \exp \left(-k t / 2 \exp \left(f_{i}\right)\right)\right|_{0} ^{\infty}=0(\operatorname{Appendix}$ A). The derivation is similar to those in Berger (1980) and Wong (2005) where above facts were assumed as conditions. Then, when $k \geq 3$, an unbiased estimator of $\mathrm{E}\left(C K L\left(f, f_{\lambda}\right)\right)$ is

$$
U B R(\lambda)=\frac{1}{n} \sum_{i=1}^{n}\left(\frac{k}{2} v\left(y_{i}\right) y_{i}^{-(k / 2-1)}+f_{\lambda i}\right) .
$$

The direct UBR estimate of $\lambda$ is the minimizer of $U B R(\lambda)$. Gaussian quadrature may be used to approximate $v\left(y_{i}\right)=\int_{0}^{y_{i}} \exp \left(-g_{\lambda i}(z)\right) z^{k / 2-1} d z$. However, it requires calculating $h_{\lambda}(i, z, \cdot)$ for several different values of $z$ which can be computationally intensive when the sample size is large. Simulations indicate that $\exp \left(g_{\lambda i}(z)\right)$ is approximately linear in $z: \exp \left(g_{\lambda i}(z)\right) \approx$ 
$\beta_{0}+\beta_{1} z$. We have tried several methods to compute the coefficients $\beta_{0}$ and $\beta_{1}$, and found that the following two work well: (1) compute $g_{\lambda i}\left(y_{i}\right)$ and $g_{\lambda i}\left(y_{i} / 2\right)$, and then compute $\beta_{0}$ and $\beta_{1}$ as the intercept and slope of the line joining two points $\left(y_{i} / 2, \exp \left(g_{\lambda i}\left(y_{i} / 2\right)\right)\right)$ and $\left(y_{i}, \exp \left(g_{\lambda i}\left(y_{i}\right)\right)\right)$; (2) from Taylor expansion $\exp \left(g_{\lambda i}(z)\right) \approx \exp \left(g_{\lambda i}\left(y_{i}\right)\right)+\left.\exp \left(g_{\lambda i}\left(y_{i}\right)\right)\left(\partial g_{\lambda i} / \partial z\right)\right|_{z=y_{i}}\left(z-y_{i}\right) \approx$ $\exp \left(f_{\lambda i}\right)+\exp \left(f_{\lambda i}\right) d_{i i}\left(z-y_{i}\right)$, where $\left.\left(\partial g_{\lambda i} / \partial z\right)\right|_{z=y_{i}} \approx d_{i i}$ based on a similar argument as that in Appendix B, and $d_{i i}$ is defined later in Section 3.3. Thus $\beta_{0} \approx \exp \left(f_{\lambda i}\right)\left(1-d_{i i} y_{i}\right)$ and $\beta_{1} \approx \exp \left(f_{\lambda i}\right) d_{i i}$. Based on the linear approximation, $v\left(y_{i}\right)$ can be calculated through recursive formulas obtained by tedious algebra (not shown). Extensive simulations indicate that above linear approximations lead to similar UBR estimates of the smoothing parameters as those based on the Gaussian quadrature, and two methods for computing the coefficients $\beta_{0}$ and $\beta_{1}$ also lead to similar UBR estimates. We will use the linear approximation based on the Taylor expansion in our simulations since it requires the least amount of computation.

\subsection{Generalized Approximate Cross Validation Method}

Let $f_{\lambda}^{(-i)}$ be the minimizer of (5) without the $i$ th observation and $f_{\lambda i}^{(-i)}=f_{\lambda}^{(-i)}\left(x_{i}\right)$. Replacing $\exp \left(f_{i}-f_{\lambda i}\right)$ by $y_{i} \exp \left(-f_{\lambda i}^{(-i)}\right)$ and ignoring the multiplying constant $1 / n$, we obtain a crossvalidation estimate of $C K L\left(f, f_{\lambda}\right)$

$$
C V(\lambda)=\sum_{i=1}^{n}\left(y_{i} \exp \left(-f_{\lambda i}^{(-i)}\right)+f_{\lambda i}\right) .
$$

It is usually expensive to compute $C V(\lambda)$ for large $n$. We now introduce an approximation of $C V(\lambda)$. Let $\boldsymbol{f}=\left(f_{1}, \cdots, f_{n}\right)^{T}, \boldsymbol{T}_{n \times m}=\left\{\phi_{v}\left(x_{i}\right)\right\}_{i=1 v=1}^{n}$ and $\boldsymbol{\Sigma}=\left\{R_{1}\left(x_{i}, x_{j}\right)\right\}_{i, j=1}^{n}$. Let $\boldsymbol{T}=\left(\boldsymbol{Q}_{1} \boldsymbol{Q}_{2}\right)\left(\boldsymbol{R}^{T} \mathbf{0}^{T}\right)^{T}$ be the QR decomposition of $\boldsymbol{T}$ and $\boldsymbol{\Omega}=\boldsymbol{Q}_{2}\left(\boldsymbol{Q}_{2}^{T} \boldsymbol{\Sigma} \boldsymbol{Q}_{2}\right)^{\dagger} \boldsymbol{Q}_{2}^{T}$ where $\dagger$ is the Moore-Penrose generalized inverse. Then $\boldsymbol{f}=\boldsymbol{T} \boldsymbol{d}+\boldsymbol{\Sigma} \boldsymbol{c}, \int_{0}^{1}\left(f^{(m)}\right)^{2} d x=\boldsymbol{f}^{T} \boldsymbol{\Omega} \boldsymbol{f}$, and the penalized likelihood (5) can be rewritten as (Xiang and Wahba 1996)

$$
J=-\sum_{i=1}^{n} l_{i}\left(f_{i}\right)+\frac{n \lambda}{2} \boldsymbol{f}^{T} \boldsymbol{\Omega} \boldsymbol{f} .
$$

Let $\boldsymbol{W}_{\lambda}=\operatorname{diag}\left(k y_{1} \exp \left(-f_{\lambda 1}\right) / 2, \cdots, k y_{n} \exp \left(-f_{\lambda n}\right) / 2\right), \boldsymbol{V}=\operatorname{diag}\left(k \exp \left(-f_{\lambda 1}\right) / 2, \cdots, k \exp \left(-f_{\lambda n}\right) / 2\right)$, and $\boldsymbol{D}=\left(\boldsymbol{W}_{\lambda}+n \lambda \boldsymbol{\Omega}\right)^{-1} \boldsymbol{V}$. An approximation of $C V(\lambda)$ is (Appendix B)

$$
A C V(\lambda)=L(\lambda)+\sum_{i=1}^{n} \frac{d_{i i} \exp \left(-f_{\lambda i}\right) y_{i}\left(y_{i}-\exp \left(f_{\lambda i}\right)\right)}{1-d_{i i} \exp \left(f_{\lambda i}\right)},
$$

where $L(\lambda)=\sum_{i=1}^{n}\left(y_{i} \exp \left(-f_{\lambda i}\right)+f_{\lambda i}\right)$ and $d_{i i}$ is the $i$ th diagnal element of $\boldsymbol{D}$. Replacing $\exp \left(f_{\lambda i}\right) d_{i i}$ by $\operatorname{tr}\left(\boldsymbol{W}_{0}^{1 / 2} \boldsymbol{D} \boldsymbol{W}_{0}^{1 / 2}\right) / n$ where $\boldsymbol{W}_{0}=\operatorname{diag}\left(\exp \left(f_{\lambda 1}\right), \cdots, \exp \left(f_{\lambda n}\right)\right)$, we get a generalized ACV

$$
G A C V_{1}(\lambda)=L(\lambda)+\frac{\operatorname{tr}\left(\boldsymbol{W}_{0}^{1 / 2} \boldsymbol{D} \boldsymbol{W}_{0}^{1 / 2}\right)}{n-\operatorname{tr}\left(\boldsymbol{W}_{0}^{1 / 2} \boldsymbol{D} \boldsymbol{W}_{0}^{1 / 2}\right)} \sum_{i=1}^{n} y_{i}\left(y_{i}-\exp \left(f_{\lambda i}\right)\right) \exp \left(-2 f_{\lambda i}\right) .
$$


Replacing $\exp \left(f_{\lambda i}\right) d_{i i}$ in the denominator by $\operatorname{tr}\left(\boldsymbol{W}_{0}^{1 / 2} \boldsymbol{D} \boldsymbol{W}_{0}^{1 / 2}\right) / n$ and $d_{i i}$ in the numerator by $\operatorname{tr}(\boldsymbol{D}) / n$, we get another generalized ACV

$$
G A C V_{2}(\lambda)=L(\lambda)+\frac{\operatorname{tr}(\boldsymbol{D})}{n-\operatorname{tr}\left(\boldsymbol{W}_{0}^{1 / 2} \boldsymbol{D} \boldsymbol{W}_{0}^{1 / 2}\right)} \sum_{i=1}^{n} y_{i}\left(y_{i}-\exp \left(f_{\lambda i}\right)\right) \exp \left(-f_{\lambda i}\right) .
$$

$G A C V_{2}(\lambda)$ is the same as the $G A C V$ in Yuan and Wahba (2004) (note that there is a typo in their formula). The GACV1 and GACV2 estimates of $\lambda$ are minimizers of $G A C V_{1}(\lambda)$ and $G A C V_{2}(\lambda)$ respectively.

\subsection{Generalized Maximum Likelihood Method}

Let $F(x)=\sum_{i=1}^{M} \theta_{i} \phi_{i}(x)+b^{\frac{1}{2}} Z(x)$ be the prior for the function $f$, where $\theta_{i} \stackrel{i i d}{\sim} N(0, a)$, $b=2 / k n \lambda, Z(x)$ is a Gaussian process independent of $\theta_{i}$ 's with $E(Z(x))=0$ and $E(Z(s) Z(t))=$ $R_{1}(s, t)$. We assume that observations $\boldsymbol{y}=\left(y_{1}, \cdots, y_{n}\right)^{T}$ are generated according to the model (1) conditional on $f=F$. As $a \rightarrow \infty$, Gu (1992) showed that the posterior mean $\mathrm{E}(F(x) \mid \boldsymbol{y})$ approximately equals the spline estimate $f_{\lambda}(x)$.

Let $u_{i c}$ and $w_{i c}$ be $u_{i}$ and $w_{i}$ evaluated at convergence of the Newton procedure. Let $\boldsymbol{u}_{c}=\left(u_{1 c}, \cdots, u_{n c}\right)^{T}, \boldsymbol{W}_{c}=\operatorname{diag}\left(w_{1 c}, \cdots, w_{n c}\right), \boldsymbol{f}_{\lambda}=\left(f_{\lambda 1}, \cdots, f_{\lambda n}\right)^{T}, \boldsymbol{y}_{c}=\boldsymbol{f}_{\lambda}-\boldsymbol{W}_{c}^{-1} \boldsymbol{u}_{c}$, $\boldsymbol{\Sigma}_{c}=\boldsymbol{W}_{c}^{1 / 2} \boldsymbol{\Sigma} \boldsymbol{W}_{c}^{1 / 2}$ and $\boldsymbol{T}_{c}=\boldsymbol{W}_{c}^{1 / 2} \boldsymbol{T}$. Let $\left(\boldsymbol{Q}_{1 c} \boldsymbol{Q}_{2 c}\right)\left(\boldsymbol{R}_{c}^{T} \mathbf{0}^{T}\right)^{T}$ be the QR decomposition of $\boldsymbol{T}_{c}$, and $\boldsymbol{U} \boldsymbol{\Lambda} \boldsymbol{U}^{T}$ be the spectral decomposition of $\boldsymbol{Q}_{2 c}^{T} \boldsymbol{\Sigma}_{c} \boldsymbol{Q}_{2 c}$ where $\boldsymbol{\Lambda}=\operatorname{diag}\left(\lambda_{\nu n}, \nu=1, \cdots, n-m\right)$. Let $\mathbf{z}=\left(z_{1}, \cdots, z_{n-m}\right)^{T}=\boldsymbol{U}^{T} \boldsymbol{Q}_{2 c}^{T} \boldsymbol{W}_{c}^{1 / 2} \boldsymbol{y}_{c}$. Then, using the equation (16) in Liu, Meiring and Wang (2005) and ignoring a constant, we have an approximation of the negative log marginal likelihood of $\boldsymbol{y}$

$$
G M L(\lambda)=-\sum_{i=1}^{n} l_{i}\left(f_{\lambda i}\right)-\frac{k}{4} \boldsymbol{u}_{c}^{T} \boldsymbol{W}_{c}^{-1} \boldsymbol{u}_{c}+\frac{1}{2} \sum_{\nu=1}^{n-m}\left(\ln \left(\lambda_{\nu n} / n \lambda+1\right)+\frac{k z_{\nu}^{2} / 2}{\lambda_{\nu n} / n \lambda+1}\right)+\ln \left|\boldsymbol{R}_{c}\right| .
$$

The GML estimate of $\lambda$ is the minimizer of $G M L(\lambda)$. Liu et al. (2005) used the approximated marginal likelihood to construct GML tests for generalized linear models. This approximation has not been explored as a tool for selecting the smoothing parameter for non-Gaussian data. Its performance is unknown.

\section{SIMULATIONS}

One simple and commonly used approach is to transform $y_{i}$ in the model (1) using logarithm and then fit the transformed data using a penalized least square smoothing spline. We refer to this simple approach as the transformation method. In the following simulation, we use the GCV method to select the smoothing parameter for the transformation approach.

We generate data from the model (1) with $f(x)=2 \sin (2 w x)+3$ and $x_{i}=i / n$. We use a factorial design with three different frequencies $w=1,2,3$, four different sample sizes 
$n=100,200,300,400$, and four different degrees of freedom $k=1,2,3,4$. We fit the model (1) using eight methods: direct UBR, direct GML, GACV1, GACV2, indirect UBR, indirect GCV, indirect GML and transformation. For each simulation setting, we repeat the simulation 100 times.

Table 1 lists the number of replications that the indirect methods failed to converge. Convergence is achieved by all other methods when $k=1,2,3$, and by all methods when $k=4$. Contrary to our experience with the Binomial and Poisson data, the indirect methods sometimes fail to converge. The non-convergence problem is quite severe for the indirect UBR method when $k=1$ and disappears quickly as $k$ increases. When $k=1$, the problem remains even when the sample size is large $(n=400)$.

$$
\text { Put Table } 1 \text { here }
$$

Figure 1 shows the CKL for all settings. In general, direct methods perform better than indirect methods. The indirect UBR method suffers from the non-convergence problem. When converges, the indirect UBR method performs as well as the direct UBR method. In additional to the occasional non-convergence problem, the indirect GCV and GML methods have larger CKL than their direct counterparts. The simple transformation method has much larger CKL especially when $k$ is very small ( $k=1$ and $k=2$ ).

Among the direct methods, UBR and GML always have the best performance. Therefore, they are recommended. GACV2 performs better than GACV1, neither of which performs as well as the direct UBR and GML methods due to occasional over-smoothing. Even though the unbiasedness property is established for $k \geq 3$ only, we nevertheless applied the direct UBR method to the cases of $k=1$ and $k=2$. Performances of the direct UBR method for these cases are similar to those for larger $k$. The direct GML method is new for non-Gaussian data. We postulate that it can be extended to Binomial and Poisson data, and can perform better than some existing methods.

\section{Put Figure 1 here}

\section{APPLICATION}

Comparative genomic hybridization $(\mathrm{CGH})$ is a technique designed for detecting segmental genomic alterations. Recent advances in array-based CGH (aCGH) technology have enabled examination of chromosomal regions in unprecedented detail, revolutionizing our understanding of chromosome alterations associated with tumorigenesis and many developmental abnormalities (Albertson and Pinkel 2003, Mantripragada, Buckley, de Stahl and Dumanski 2004). The aCGH technology uses two differentially labeled test and reference DNAs which are cohybridized to cloned genomic fragments immobilized on glass slides. The hybridized DNAs are then de- 
tected in two different fluorochromes, and the significant deviation from unity in the ratios of the digitized intensity values is indicative of copy-number differences between the test and reference genomes (Wang and Guo 2004). Accurate identification of amplified or deleted regions requires estimates of variances (Moore, Pallavicini, Cher and Gray 1997, Wang and Guo 2004). The number of replicated arrays is typically small owing to the cost. Therefore, the standard sample variance estimates are unreliable and some simple improvements have been proposed (Moore et al. 1997, Tusher, Tibshirani and Chu 2001, Huang and Pan 2002, Wang and Guo 2004). Wang and Guo (2004) considered the variance as a function of the physical locations in the genome. They applied the lowess smoother to the logarithm of sample variances with a fixed smoothing parameter. They have shown that using the smoothed variances in the t-test can lead to large gains in the power. As we have shown in Section 4, the simple approach to smoothing the logarithm of sample variances is less efficient when the number of replications is small. Also, the selection of the smoothing parameter in Wang and Guo (2004) is somewhat arbitray.

To illustrate our new methods, we downloaded a well-known BAC array data sets from the website http://www.nature.com/ng/journal/v29/n3/suppinfo/ng754_S1.html. The data result from an experiment aimed at measuring copy number changes for the cell strains (test samples) against normal male reference DNAs (references), which were co-hybridized on CGH arrays containing 2,460 BAC and P1 clones in triplicate (7,380 spots) (Snijders, Nowak, Segraves, Brown, Conroy, Hamilton, Hindle, Huey, Kimura, Law, Myambo, Palmer, Ylstra, Yue, Gray, Jain, Pinkel and Albertson 2001).

For each chromosome of a cell strain, we assume model (2) where $z_{i j}$ is the $j$ th replicate of $\log _{2}$ fluorescent ratio of clone $i, \mu_{i}$ is the mean fluorescent ratio, $x_{i}$ is the physical position along the chromosome, and $k=2$. A non-zero $\mu_{i}$ corresponds to an alteration. Wang and Guo (2004) introduced a smoothed t-statistic with variances smoothed along the genome. They have shown that the smoothed t-statistic always improves the performance over the standard t-statistic. We focus on estimation of the variance function. For simplicity we transform the genome position $x_{i}$ into the interval $[0,1]$.

We fitted variance functions to all chromosomes of all cell strains. Figure 2 shows observations, fits and confidence intervals for chromosome 10 of the cell strain GM13330010220, and chromosomes 10 and 11 of the cell strain GM03134001218. For the lowess fits, as in Wang and Guo (2004), we used $30 \%$ of the data for smoothing at each position. For the smoothing spline fit, we used the direct GML method to select the smoothing parameter. The approximate Bayesian confidence intervals were computed using the methods in Wahba et al. (1995). Some variances are relatively large and some variances are very small (Figure 2). Replacing variances by their smoothed estimates also reduces the effect of outliers and prevents clones with very small variances from dominating the result. Other approaches have been proposed to deal with 
very small variances (Tusher et al. 2001).

Put Figure 2 here

\section{APPENDIX A}

We will show that when $k \geq 3$, (a) $\left.v(t) \exp \left(-k t / 2 \exp \left(f_{i}\right)\right)\right|_{0} ^{\infty}=0$, and (b) $\mathrm{E}\left(\exp \left(-f_{\lambda i}\right)\right)<$ $\infty$.

For (a), we will show that $\lim _{t \rightarrow 0} v(t)=0$ and $\lim _{t \rightarrow \infty}\left(v(t) \exp \left(-k t / 2 \exp \left(f_{i}\right)\right)\right)=0$. We will use the following fact that for any $a>0$ and $x$,

$$
a \exp (-x)+x \geq 1+\ln a
$$

Above inequality can be verified by finding the minimum of the left hand side as a function of $x$.

Note that $\boldsymbol{y}_{-i}$ is fixed and the $i$ th observation is replaced by $z$. The penalized likelihood (5) is equivalent to

$$
I(f)=z \exp \left(-f_{i}\right)+f_{i}+\sum_{j \neq i}^{n}\left(y_{j} \exp \left(-f_{j}\right)+f_{j}\right)+\frac{n \lambda}{k} \int_{0}^{1}\left(f^{(m)}\right)^{2} d x .
$$

Note that $h_{\lambda}(i, z, \cdot)$ is the minimizer of $(18)$ and $g_{\lambda i}(z)=h_{\lambda}\left(i, z, x_{i}\right)$.

We assume that the constant function, $f_{0}(x)=0$, belongs to the model space. This is true when the model space is $W_{m}([0,1])$. $I\left(f_{0}\right)=z+\sum_{j \neq i} y_{j}$. Since $h_{\lambda}(i, t, \cdot)$ is the minimizer of $I(f)$, we have

$$
\begin{aligned}
z \exp \left(-g_{\lambda i}(z)\right)+g_{\lambda i}(z) & \leq I\left(h_{\lambda}(i, z, \cdot)\right)-\sum_{j \neq i}\left(y_{j} \exp \left(-h_{\lambda}\left(i, z, x_{j}\right)\right)+h_{\lambda}\left(i, z, x_{j}\right)\right) \\
& \leq I\left(f_{0}\right)-\sum_{j \neq i}\left(y_{j} \exp \left(-h_{\lambda}\left(i, z, x_{j}\right)\right)+h_{\lambda}\left(i, z, x_{j}\right)\right) \\
& \leq z+\sum_{j \neq i} y_{j}-\sum_{j \neq i}\left(1+\ln y_{j}\right),
\end{aligned}
$$

where the last inequality is based on (17). Therefore, as $z \rightarrow 0, z \exp \left(-g_{\lambda i}(z)\right)+g_{\lambda i}(z)$ is bounded above.

We claim that for any power $p>1$,

$$
\lim _{z \rightarrow 0} z^{p} \exp \left(-g_{\lambda i}(z)\right)=0
$$

Otherwise, there exists some $p_{1}>1$ such that $\limsup _{z \rightarrow 0} z^{p_{1}} \exp \left(-g_{\lambda i}(z)\right)>0$. Let $p_{2}=\left(p_{1}+1\right) / 2$. Then $\limsup _{z \rightarrow 0} z^{p_{2}} \exp \left(-g_{\lambda i}(z)\right)=\infty$. There exists a sequence $z_{m} \rightarrow 0$ such that $-g_{\lambda i}\left(z_{m}\right)+$ 
$p_{2} \ln z_{m} \rightarrow \infty$. Also, $-g_{\lambda i}\left(z_{m}\right)+\ln z_{m} \geq-g_{\lambda i}\left(z_{m}\right)+p_{2} \ln z_{m} \rightarrow \infty$. Therefore, for large enough $m$, we have $\left(-g_{\lambda i}\left(z_{m}\right)+\ln z_{m}\right) / 2 \geq 1 /\left(p_{2}-1\right)>0$. Thus, for large enough $m$,

$$
\begin{aligned}
z_{m} \exp \left(-g_{\lambda i}\left(z_{m}\right)\right)+g_{\lambda i}\left(z_{m}\right) & =\exp \left(-g_{\lambda i}\left(z_{m}\right)+\ln z_{m}\right)+g_{\lambda i}\left(z_{m}\right) \\
& \geq 1-g_{\lambda i}\left(z_{m}\right)+\ln z_{m}+\left(-g_{\lambda i}\left(z_{m}\right)+\ln z_{m}\right)^{2} / 2+g_{\lambda i}\left(z_{m}\right) \\
& \geq \ln z_{m}+\left(-g_{\lambda i}\left(z_{m}\right)+\ln z_{m}\right) /\left(p_{2}-1\right) \\
& =\left(-g_{\lambda i}\left(z_{m}\right)+p_{2} \ln z_{m}\right) /\left(p_{2}-1\right) .
\end{aligned}
$$

The left hand side is bounded above while the right hand side approaches to $\infty$, a contradiction.

Taking $p=1.1$ in (20), when $k \geq 3$, we have

$$
\limsup _{t \rightarrow 0} v(t)=\limsup _{t \rightarrow 0} \int_{0}^{t}\left(z^{1.1} \exp \left(-g_{\lambda i}(z)\right)\right) z^{\frac{k}{2}-2.1} d z \leq \limsup _{t \rightarrow 0} \int_{0}^{t} z^{\frac{k}{2}-2.1} d z=0 .
$$

Therefore, $\lim _{t \rightarrow 0} v(t)=0$ since $v(t) \geq 0$.

As $z \rightarrow \infty$, from (19), we have $z \exp \left(-g_{\lambda i}(z)\right)+g_{\lambda i}(z) \leq 2 z$. On the other hand, from (17) with $a=z / 2,-g_{\lambda i}(z) \leq z \exp \left(-g_{\lambda i}(z)\right) / 2$ when $z \geq 2$. Therefore, $z \exp \left(-g_{\lambda i}(z)\right) \leq$ $2 z-g_{\lambda i}(z) \leq 2 z+z \exp \left(-g_{\lambda i}(z)\right) / 2$. There exists some large $T$ such that

$$
\exp \left(-g_{\lambda i}(z)\right) \leq 4, \quad z \geq T
$$

Now,

$$
v(t)=\int_{0}^{T} \exp \left(-g_{\lambda i}(z)\right) z^{\frac{k}{2}-1} d z+\int_{T}^{t} \exp \left(-g_{\lambda i}(z)\right) z^{\frac{k}{2}-1} d z \leq v(T)+\frac{8}{k} t^{\frac{k}{2}}
$$

Then

$$
\limsup _{t \rightarrow \infty}\left(v(t) \exp \left(\frac{-k t}{2 \exp \left(f_{i}\right)}\right)\right) \leq \limsup _{t \rightarrow \infty}\left(v(T)+\frac{8}{k} t^{\frac{k}{2}}\right) \exp \left(\frac{-k t}{2 \exp \left(f_{i}\right)}\right)=0 .
$$

Therefore, $\lim _{t \rightarrow \infty}\left(v(t) \exp \left(-k t / 2 \exp \left(f_{i}\right)\right)\right)=0$.

Now we prove (b) $\mathrm{E}\left(\exp \left(-f_{\lambda i}\right)\right)<\infty$. From (20), when $k \geq 3$, there exist a small enough $\epsilon_{1}$ such that $\mathrm{E}\left(\exp \left(-f_{\lambda i}\right) I_{\left\{y_{i}<\epsilon_{1}\right\}}\right) \leq \mathrm{E}\left(y_{i}^{-1.1} I_{\left\{y_{i}<\epsilon_{1}\right\}}\right)<\infty$. Similarly, from (22), there exist a large enough $\epsilon_{2}$ such that $\mathrm{E}\left(\exp \left(-f_{\lambda i}\right) I_{\left\{y_{i}>\epsilon_{2}\right\}}\right)<\infty$. Then $\mathrm{E}\left(\exp \left(-f_{\lambda i}\right)\right)=\mathrm{E}\left(\exp \left(-f_{\lambda i}\right) I_{\left\{y_{i}<\epsilon_{1}\right\}}\right)+$ $\mathrm{E}\left(\exp \left(-f_{\lambda i}\right) I_{\left\{y_{i}>\epsilon_{2}\right\}}\right)+\mathrm{E}\left(\exp \left(-f_{\lambda i}\right) I_{\left\{\epsilon_{1} \leq y_{i} \leq \epsilon_{2}\right\}}\right)<\infty$.

\section{APPENDIX B}

Lemma 1. (Leaving-out-one lemma) $h_{\lambda}\left(i, \exp \left(f_{\lambda i}^{(-i)}\right), x\right)=f_{\lambda}^{(-i)}(x)$.

The proof is omitted since it is similar to that in Xiang and Wahba (1996). Lemma 1 states that the estimated function based on $\boldsymbol{y}^{-i}=\left(y_{1}, \cdots, y_{i-1}, \exp \left(f_{\lambda i}^{(-i)}\right), y_{i+1}, \cdots, y_{n}\right)^{T}$ is the same as $f_{\lambda}^{(-i)}$. It is easy to check that $\partial^{2} J / \partial \boldsymbol{f} \partial \boldsymbol{f}^{T}=\boldsymbol{W}_{\lambda}+n \lambda \boldsymbol{\Omega}$ and $\partial^{2} J / \partial \boldsymbol{y} \partial \boldsymbol{f}^{T}=-\boldsymbol{V}$. Using Taylor expansions as in Xiang and Wahba (1996), we have $\left(f_{\lambda i}-f_{\lambda i}^{(-i)}\right) /\left(y_{i}-\exp \left(f_{\lambda i}^{(-i)}\right)\right) \approx d_{i i}$. Thus by (14), 


$$
\begin{aligned}
C V(\lambda) & =L(\lambda)+\sum_{i=1}^{n} y_{i}\left(\exp \left(-f_{\lambda i}^{(-i)}\right)-\exp \left(-f_{\lambda i}\right)\right) \\
& \approx L(\lambda)+\sum_{i=1}^{n} y_{i} \exp \left(-f_{\lambda i}\right)\left(f_{\lambda i}-f_{\lambda i}^{(-i)}\right) \\
& =L(\lambda)+\sum_{i=1}^{n} y_{i} \exp \left(-f_{\lambda i}\right) \frac{f_{\lambda i}-f_{\lambda i}^{(-i)}}{y_{i}-\exp \left(f_{\lambda i}^{(-i)}\right)} \frac{y_{i}-\exp \left(f_{\lambda i}\right)}{1-\frac{\exp \left(f_{\lambda i}\right)-\exp \left(f_{\lambda i}^{(-i)}\right)}{y_{i}-\exp \left(f_{\lambda i}^{(-i)}\right)}} \\
& \approx L(\lambda)+\sum_{i=1}^{n} y_{i} \exp \left(-f_{\lambda i}\right) \frac{f_{\lambda i}-f_{\lambda i}^{(-i)}}{y_{i}-\exp \left(f_{\lambda i}^{(-i)}\right)} \frac{y_{i}-\exp \left(f_{\lambda i}\right)}{1-\exp \left(f_{\lambda i}\right) \frac{f_{\lambda i}-f_{\lambda i}^{(-i)}}{y_{i}-\exp \left(f_{\lambda i}^{(-i)}\right)}} \\
& =L(\lambda)+\sum_{i=1}^{n} \frac{y_{i} \exp \left(-f_{\lambda i}\right)\left(y_{i}-\exp \left(f_{\lambda i}\right)\right)}{\frac{y_{i}-\exp \left(f_{\lambda i}^{(-i)}\right)}{f_{\lambda i}-f_{\lambda i}^{(-i)}}-\exp \left(f_{\lambda i}\right)} \\
& \approx L(\lambda)+\sum_{i=1}^{n} \frac{d_{i i} y_{i} \exp \left(-f_{\lambda i}\right)\left(y_{i}-\exp \left(f_{\lambda i}\right)\right)}{1-d_{i i} \exp \left(f_{\lambda i}\right)}
\end{aligned}
$$

\section{References}

Albertson, D. G. and Pinkel, D. (2003). Genomic microarray in human genetic disease and cancer, Human Molecular Genetics 12: R145-R152.

Andersen, T. G. and Lund, J. (1997). Estimating continuous-time stochastic volatility models of the short-term interest rate, Journal of Econometrics 77: 343-377.

Berger, J. O. (1980). Improving on inadmissible estimators in continuous exponential families with applications to simultaneous estimation of gamma scale parameters, Annals of Statistics 8: 545-571.

Box, G. (1988). Signal-to-noise ratios, performance criteria, and transformations, Technometrics 30: 1-17.

Carroll, R. J. (1982). Adapting for heteroscedasticity in linear models, The Annals of Statistics 10: $1224-1233$.

Carroll, R. J. (1987). The effect of variance function estimation on prediction intervals, Proc. 4th Purdue Symp. Statistical Decision Theory and Related Topics, Eds J. O. Berger and S. S. Gupta, Vol II.

Carroll, R. J. and Ruppert, D. (1988). Transformation and weighting in regression, Chapman \& Hall Ltd. 
Cui, X., Hwang, J. T. G., Qiu, J., Blades, N. J. and Churchill, G. A. (2005). Improved statistical tests for differential gene expression by shrinking variance components estimates, Biostatistics 6: 59-75.

Dai, M. and Guo, W. (2005). Heteroscedastic smoothing spline models, Unpublished Manuscript.

Fan, J. and Yao, Q. (1998). Efficient estimation of conditional variance functions in stochastic regression, Biometrika 85: 645-660.

Gallant, A. R. and Tauchen, G. (1997). Estimation of continuous time models for stock returns and interest rates, Macroecon. Dyn. 1: 135-168.

Gu, C. (1992). Cross-validating non Gaussian data, Journal of Computational and Graphical Statistics 2: 169-179.

Gu, C. (2002). Smoothing Spline ANOVA Models, Springer-Verlag, New York.

Gu, C. and Xiang, D. (2001). Cross-validating Non-Gaussian data: Generalized approximate cross-validation revisited, Journal of Computational and Graphical Statistics 10(3): 581591.

Hall, P. and Carroll, R. J. (1989). Variance function estimation in regression: The effect of estimating the mean, Journal of the Royal Statistical Society, Series B, Methodological 51: $3-14$.

Huang, X. and Pan, W. (2002). Comparing three methods for variance estimation with duplicated high density oligonucleotide arrays, Funct Integr Genomics 2: 126-133.

Liu, A., Meiring, W. and Wang, Y. (2005). Testing generalized linear models using smoothing spline methods, Statistica Sinica 15: 235-256.

Mantripragada, K. K., Buckley, P. G., de Stahl, T. D. and Dumanski, J. P. (2004). Genomic microarrays in the spotlight, Trends in Genetics 20: 87-94.

Moore, D. H., Pallavicini, M., Cher, M. L. and Gray, J. W. (1997). A t-statistic for objective interpretation of comparative genomic hybridization (CGH) profiles, Cytometry 28: 183190.

Müller, H. G. and Stadtmüller, U. (1987). Estimation of heteroscedasticity in regression analysis, Annals of Statistics 15: 610-635.

Raab, G. M. (1981). Estimation of a variance function, with application to immunoassay, Applied Statistics 30: 32-40. 
Ruppert, D., Wand, M. P., Holst, U. and Hssjer, O. (1997). Local polynomial variance-function estimation, Technometrics 39: 262-273.

Silverman, B. W. (1985). Some aspects of the spline smoothing approach to non-parametric regression curve fitting (with discussion), Journal of the Royal Statistical Society, Series B, Methodological 47: 1-21.

Snijders, A. M., Nowak, N., Segraves, R. Blackwood, S., Brown, N., Conroy, J., Hamilton, G., Hindle, A. K., Huey, B., Kimura, K., Law, S., Myambo, K., Palmer, J., Ylstra, B., Yue, J. P., Gray, J. W., Jain, A. N., Pinkel, D. and Albertson, D. G. (2001). Assembly of microarrays for genome-wide measurement of DNA copy number, Nature Genetics 29: 263264.

Tusher, V., Tibshirani, R. and Chu, G. (2001). Significance analysis of microarrays applied to the ionizing radiation response, Proc Natl Acad Sci 98: 5116-5121.

Wahba, G., Wang, Y., Gu, C., Klein, R. and Klein, B. (1995). Smoothing spline ANOVA for exponential families, with application to the Wisconsin Epidemiological Study of Diabetic Retinopathy, Annals of Statistics 23: 1865-1895.

Wang, Y. and Guo, S. W. (2004). Statistical methods for detecting genomic alterations through array-based comparative genomic hybridization (CGH), Frontiers in Bioscience 9: 540549.

Wang, Y., Wahba, G., Chappell, R. and Gu, C. (1995). Simulation studies of smoothing parameter estimates and Bayesian confidence intervals in Bernoulli SS ANOVA models, Communications in Statistics: Simulation and Computation 24: 1037-1059.

Watters, R. L., J., Carroll, R. J. and Spiegelman, C. H. (1987). Error modeling and confidence interval estimation for inductively coupled plasma calibration curves, Analytical Chemistry 59: 1639-1643.

Wong, W. (2005). Estimation of the loss of an estimate. Technical Report \#2005-18, Dept. of Statistics, University of Stanford.

Xiang, D. and Wahba, G. (1996). A generalized approximate cross validation for smoothing splines with non-gaussian data, Statistica Sinica 6: 675-692.

Yao, Q. and Tong, H. (1994). Quantifying the influence of initial values on non-linear prediction, Journal of the Royal Statistical Society, Series B, Methodological 56: 701-725.

Yuan, M. (2005). Automatic smoothing for poisson regression, Communications in StatisticsTheory and Methods 34: 603-617. 
Yuan, M. and Wahba, G. (2004). Doubly penalized likelihood estimator in heteroscedastic regression, Statistics and Probability Letters 69: 11-20. 
Table 1: Number of replications out of 100 total that failed to converge. Three numbers in each cell correspond to the indirect UBR, GML and GCV methods respectively.

\begin{tabular}{|c|c|c|c|c|c|c|c|c|c|}
\hline & \multicolumn{3}{|c|}{$w=1$} & \multicolumn{3}{c|}{$w=2$} & \multicolumn{3}{c|}{$w=3$} \\
\hline & $k=1$ & $k=2$ & $k=3$ & $k=1$ & $k=2$ & $k=3$ & $k=1$ & $k=2$ & $k=3$ \\
\hline$n=100$ & $98,0,0$ & $19,1,0$ & $0,0,0$ & $100,0,0$ & $37,4,2$ & $0,0,0$ & $100,0,0$ & $34,0,0$ & $1,0,0$ \\
$n=200$ & $97,0,0$ & $8,0,2$ & $0,0,0$ & $100,0,0$ & $15,2,0$ & $0,0,0$ & $100,0,0$ & $12,0,0$ & $0,0,0$ \\
$n=300$ & $92,0,0$ & $4,0,0$ & $0,0,0$ & $100,0,0$ & $6,0,0$ & $0,0,0$ & $100,0,0$ & $4,0,0$ & $0,0,0$ \\
$n=400$ & $88,0,0$ & $2,1,0$ & $0,0,0$ & $100,0,0$ & $2,1,0$ & $0,0,0$ & $100,0,0$ & $1,0,0$ & $0,0,0$ \\
\hline
\end{tabular}




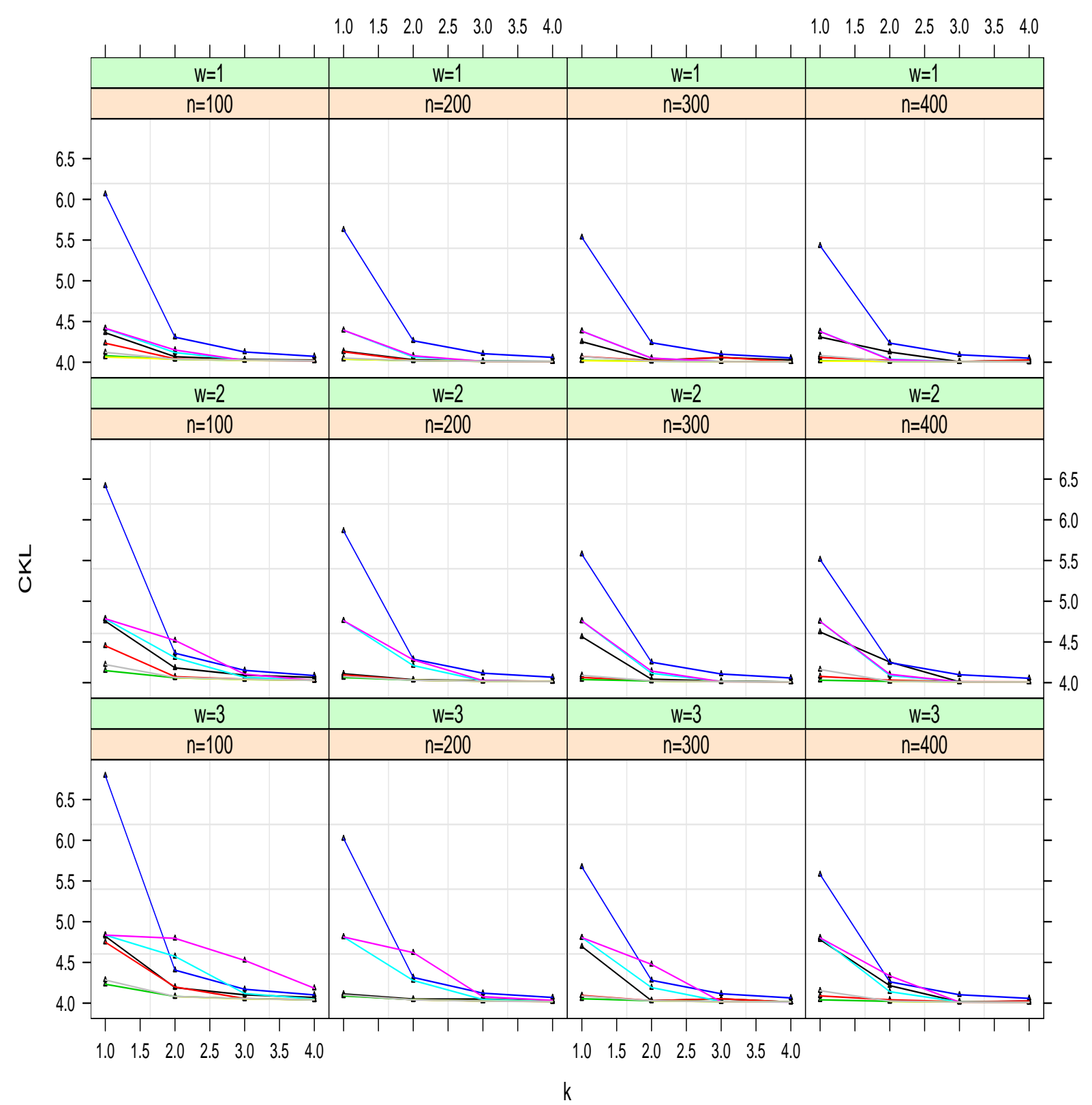

Figure 1: Plots of CKL with colored lines representing the direct UBR (gray), direct GML (green), GACV1 (black), GACV2 (red), indirect URB (yellow), indirect GCV (light blue), indirect GML (pink) and transformation (blue) methods. 

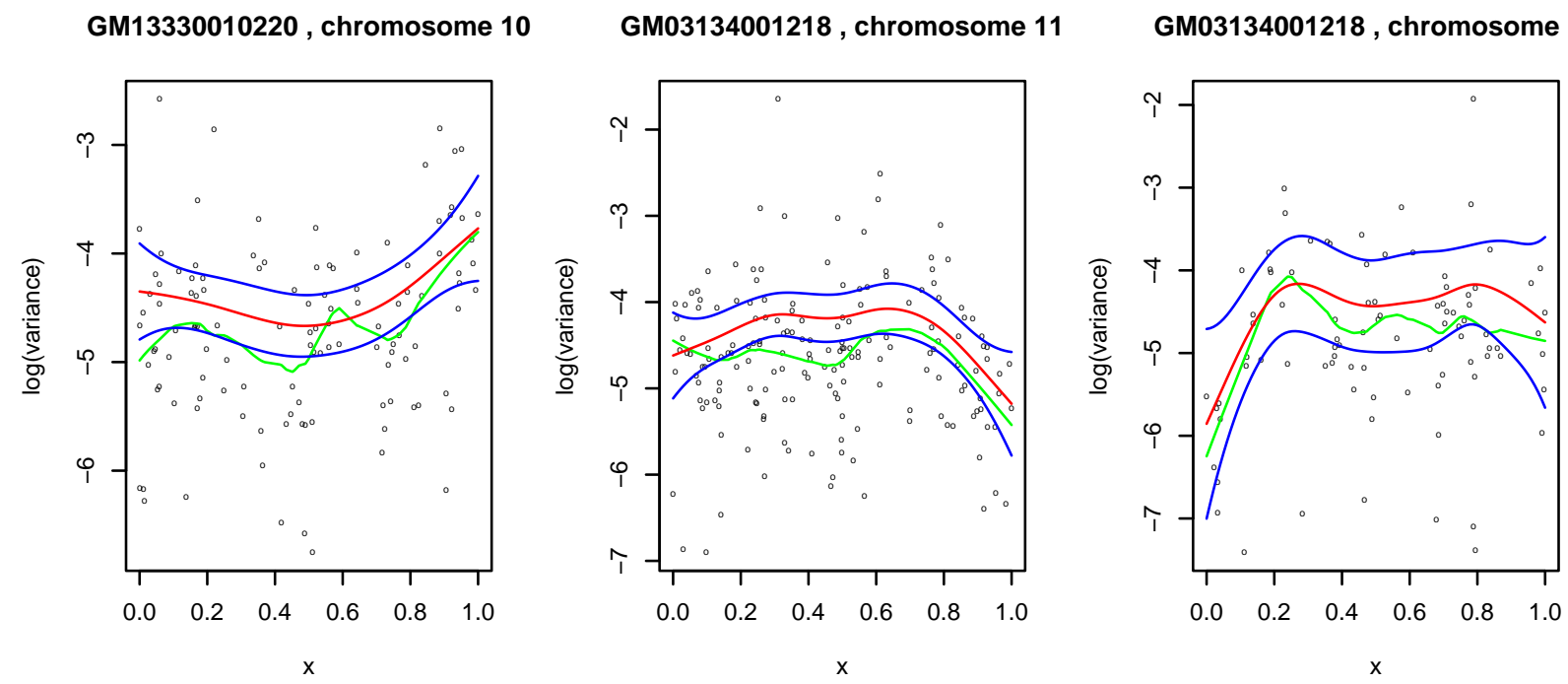

Figure 2: Plots of observations and fits for chromosome 10 of the cell strain GM13330010220 (left), and chromosomes 10 (middle) and 11 (right) of the cell strain GM03134001218. Circles are sample variances on logarithm scale. Green and red lines are lowess fits and smoothing spline fits respectively. Two blue lines in each plot are 95\% Bayesian confidence intervals of the smoothing spline fit. 